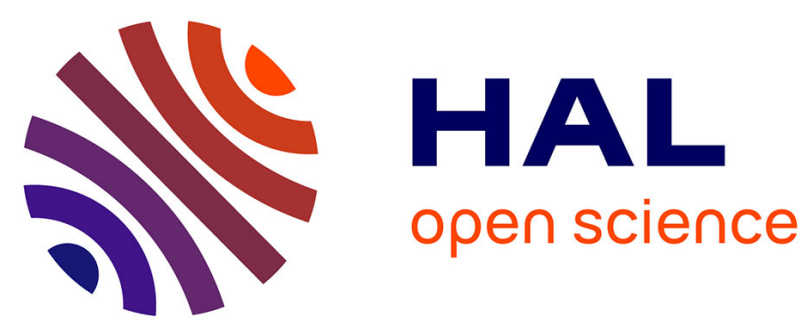

\title{
Multi-sensor Array Based Fire Monitor for Cotton Pile
}

Chenrui Bai, Junning Zhang, Chengxu Lv, Liguo Wei, Liming Zhou, Bo Zhao

\section{To cite this version:}

Chenrui Bai, Junning Zhang, Chengxu Lv, Liguo Wei, Liming Zhou, et al.. Multi-sensor Array Based Fire Monitor for Cotton Pile. 11th International Conference on Computer and Computing Technologies in Agriculture (CCTA), Aug 2017, Jilin, China. pp.540-553, 10.1007/978-3-030-061371_50. hal-02124226

\section{HAL Id: hal-02124226 \\ https://hal.inria.fr/hal-02124226}

Submitted on 9 May 2019

HAL is a multi-disciplinary open access archive for the deposit and dissemination of scientific research documents, whether they are published or not. The documents may come from teaching and research institutions in France or abroad, or from public or private research centers.
L'archive ouverte pluridisciplinaire HAL, est destinée au dépôt et à la diffusion de documents scientifiques de niveau recherche, publiés ou non, émanant des établissements d'enseignement et de recherche français ou étrangers, des laboratoires publics ou privés.

\section{(c)(1)}

Distributed under a Creative Commons Attribution| 4.0 International License 


\title{
Multi-sensor Array based Fire Monitor for Cotton
}

\section{Pile}

\author{
Chenrui Bai, Junning Zhang ${ }^{(凶)}$, Chengxu Lv, Liguo Wei, Liming Zhou, Bo \\ Zhao
}

Chinese Academy of Agricultural Mechanization Sciences, Beijing 100083, China

bcr7048190@163.com

\begin{abstract}
Cotton is a strategic material for people's livelihood, however, its fiber is most combustible in all kinds of natural fiber. To warn this risk, the mechanism of cotton pile smoke and fire was studied in this paper, and the monitoring and early warning indicators of the cotton pile fire were selected. The multi-sensor array was designed and to developed sense temperature, humidity and CO concentration. The fire monitoring device has the key modules of temperature sensor, humidity sensor, CO gas sensor, and PC center. A user interface was developed on LabVIEW to display and control data. Multi-sensor array based fire monitor can provide the data of temperature, humidity and CO concentration. It is essential to effectively measure abnormal or normal situation of the cotton pile, and can make early warning for the cotton fire and reduce the loss of cotton fire disaster.
\end{abstract}

Keywords: Unginned cotton smoldering; Temperature ; Humidity; CO concentration; Fire warning

\section{Introduction}

Cotton is a strategic commodity for the people's livelihood, it is involved in two major goods industries of agriculture and textile industry. Cotton closely related to people's lives, it is known as the white coal, and its value can be comparable to energy ore [1]. At the same time, cotton fiber is the most combustible in all kinds of natural fibers. It is easy to burn at the oxygen index of 18 19 and ignition of 160. In 
the Chinese dangerous goods category, Cotton is defined as the fourth category of "flammable solid, spontaneous combustion of goods and wet flammable materials". During the cotton post-harvest process of loading, transportation, unloading and storage, cotton smoldering could easily lead to personal injury and property damage, therefore, it's important to ensure cotton safety. This is conducive to the rapid development of the cotton industry. In particular, the cotton machinery harvest technology of China is growing rapidly, so cotton storage safety during the processing is serious [2]. The impurities rate and moisture regain of the cotton harvested by machine is higher than that picked by hand. During the interval between harvest and processing, the cotton usually need to be stored in the yard for a period of time. Because of the internal temperature and humidity, the cotton pile is easily fired [3]. This not only puts forward the new request to the processing technology, but also has the urgent technical demand on fire early warning during storage.

At present, the combustion characteristics of fiber fabrics have been researched, but burning characteristics of cotton, which focuses on the cotton smoldering characteristics, need to be further studied. Wakelyn P J et al. presented the cotton physicochemical properties, classification, production and application[4]. Bjarne C. Hagen et al. studied the effect of heat flux on cotton mullion, showing the result that both heat flux and material density affect smoldering temperature [5]. At the same time, the effects of cotton density has been studied as well, the experiments and consistent theoretical calculations showed that an increasing density leads to ignition temperature decrease. Density affects both the ignition temperatures and the mass loss rates in a systematic way [6]. Chang $\mathrm{L}$ et al. conducted both smoldering and thermal analysis experiments to study the smoldering characteristics of the maintenance and exotherm. The results showed that smoldering was just selfsustained at the sample width of $10 \mathrm{~cm}$, it typically propagated to the end in natural convection. When sample width was less than $10 \mathrm{~cm}$, smoldering gradually quenched after ignited by cigarette, otherwise, it propagated forwards with peak temperature gradually promoting to higher value, and even transited from smoldering to flaming [7]. The harm and precautionary measures of cotton fire were studied as well. Jinjun Wang et al. studied the law of smoke spread in early stage cotton smoldering [8]. But it's lack of cotton smoldering early monitoring technology research. The cotton burning properties has been studied. For example, Qixiang Zhu expressed the temperature and humidity based cotton auto-monitor from 3 aspects of: the consequence of long-term storage, the influence of the cotton's high rate of moisture regain towards manufacturing and spinning, the relationship between the cotton quality and the temperature and humidity changing [1]. Zhang Ruoyu et al. effectively monitored the changes in the cotton temperature and humidity, and the cotton temperature and humidity monitoring system was designed based on the wireless sensor network [9].

Due to the cotton flammability characteristics, it is difficult to monitor cotton 
pile safety status and deal with the dangerous situation. Low-power and low-cost gas multi-sensor array of cotton fire monitoring system will help to effectively measure and monitor the cotton pile state. The real-time monitoring technology can be used to strictly monitor the cotton pile internal environment. The real-time data transmission is a powerful tool to take effective measures. The real-time monitoring system can save time to deal with dangerous situations, and greatly reduce the loss of fire disaster $[10,11]$.

In this paper, the mechanism of cotton pile smoke and fire was studied, and the monitoring and early warning indicators of the cotton pile fire were selected. The multi-sensor array was designed and to developed sense temperature, humidity and $\mathrm{CO}$ concentration. The fire monitoring device has the key modules of temperature sensor, humidity sensor, CO gas sensor, and PC center. A user interface was developed on LabVIEW to display and control data.

\section{Smoldering Mechanism of Unginned Cotton}

Cotton fiber oxidation and gap heat are the root of unginned cotton smoldering. The normal mature cotton fibers are composed of fluffy cellulose, and cellulose contains a large number of hydrophilic hydroxyl groups, which are puffed in water. In the environment of water vapor, cotton fiber will be oxygenized by oxidation from water vapor and release heat. At a constant temperature, more heat will be released with the increasing relative humidity and moisture content. Cotton fiber is elongated flat tube with diameter of 15-19 microns. Cotton fiber forms a natural curved cavity in the growth process and its pore takes about $20 \sim 40 \%$ volume between cavity and cotton fiber. In the state of the pores inside occlusion, cotton fiber play the role of insulating layer [12, 13].

Another cause of smoldering is the closed process of storage and transportation and the way of stacked storage. In many cotton processing enterprises, due to limited venues, unginned cotton is often piled up in the open field. There is no ventilation between the cotton pile and the cement pile, and the upper part of the pile is often covered with cloth. Thus, the internal heat of the middle and low cotton layers is difficult to disperse, and it's favorable for cotton to spontaneously combust. When the pile heat accumulates gradually, it lead to cotton spontaneous combustion. In the case of insufficient oxygen, a small amount of air in the cotton fiber pores can maintain the local or small-scale slow burning, the flame and smoke cannot be seen. It is extremely dangerous, and can last for days and not be found [1-4].

The cotton pile interior may be mildew, and the mildew cotton is gradually oxidized, causing temperature rise. Therefore, the local mildew part can finally result in a smoldering process. During this period, there is little air exchanged to the outside, so it burns incompletely, produces CO, spreading out. Conventional cotton testing equipment can only detect temperature and humidity, or moisture regain. Even though some equipment includes gas detector, it is placed inside the plant flue gas 
alarm. There is no involvement in the monitoring of the cotton pile internal smoldering gas, and ordinary factory fire alarm equipment do not raise the alarm until smoke or flame appearing. Therefore, the monitoring factors are designed in this paper, including temperature, humidity and $\mathrm{CO}$ concentration of cotton pile. The monitoring system designed in this paper sense the cotton internal temperature, humidity and smoldering gas. In the case of the abnormal temperature increase without observable fire, this system could be used for fire early warning. This system is sensitive to the increasing of temperature and CO concentration. Therefore, it could be used for fire early warning before the observable fire was found [14-20].

Table 1. The contrast of factory fire alarm system and cotton pile fire monitoring system

\begin{tabular}{lllll}
\hline & $\begin{array}{l}\text { Embeds } \\
\text { cotton pile }\end{array}$ & $\begin{array}{l}\text { Involves smoldering } \\
\text { gas monitoring }\end{array}$ & $\begin{array}{l}\text { Fire response } \\
\text { speed }\end{array}$ & $\begin{array}{l}\text { Cotton pile } \\
\text { real-time } \\
\text { monitoring }\end{array}$ \\
\hline $\begin{array}{l}\text { Factory fire alarm } \\
\text { system }\end{array}$ & No & No & Slow & No \\
$\begin{array}{l}\text { Cotton pile fire } \\
\text { monitoring system }\end{array}$ & Yes & Yes & Fast & Yes \\
\hline
\end{tabular}

\section{Fire Monitor System Development}

As figure 1 shown, multi-sensor based fire monitor system is divided into 3 modules of data sensing and acquiring module, data query and forwarding module and data receiving and display module.

According to the mechanism analysis of unginned cotton smoldering, data sensing and acquiring module needs collect 3 kinds of data of temperature, humidity and $\mathrm{CO}$ concentration, each module is connected with the CAN bus, waiting for query instructions from data query and forwarding module, and then sends the collected data to CAN bus. Data query and forwarding module queries the data on CAN bus, collects and aggregates the data. Data is packaged by PIC and send to the Zigbee transmitter through the RS232 interface. The data receiving and display module receives the data of the Zigbee receiver. The PC runs through the software interface developed by LabVIEW tools, displays the data and analysis results that have been processed by the program. 

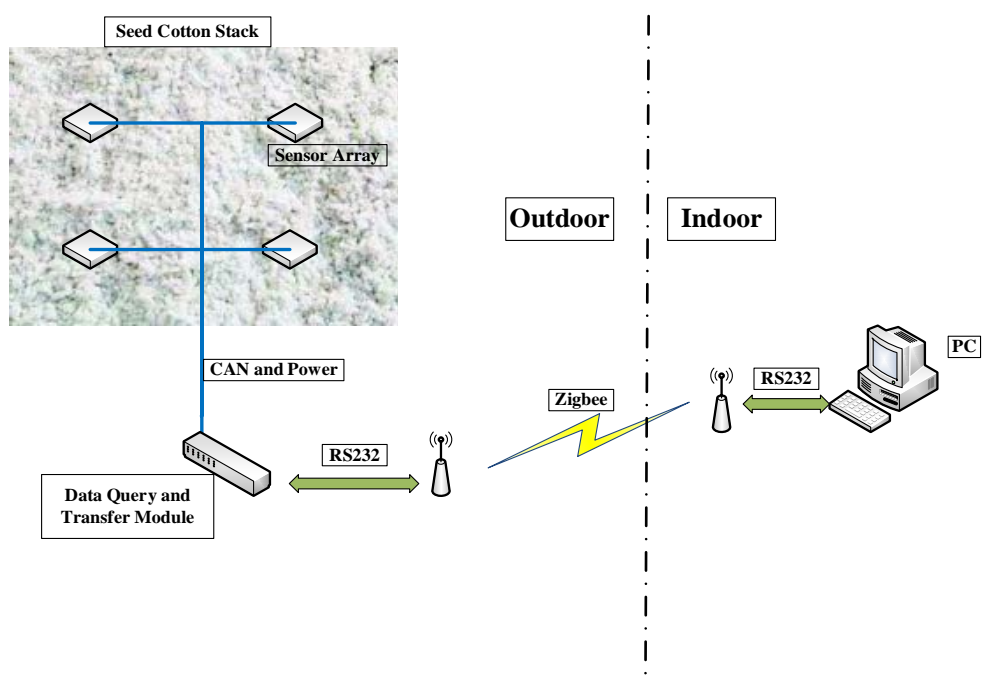

Fig. 1. Overall design

\subsection{Data Sensing and Acquiring Module}

The data sensing and acquiring module contains 2 acquisition systems of CO concentration acquisition part and temperature and humidity acquisition part.

\subsubsection{CO Concentration}

Due to the large density of cotton pile, the smoldering $\mathrm{CO}$ concentration becomes relatively low, so the LOD of selected CO sensor is low. Through the sensor working environment analysis and the accuracy requirements, British CITY 7E / F sensor was used, performing the sensitivity of $0.10 \pm 0.02 \mu \mathrm{A} / \mathrm{ppm}$. Its range and long-term stability of the signal meet all above requirements.

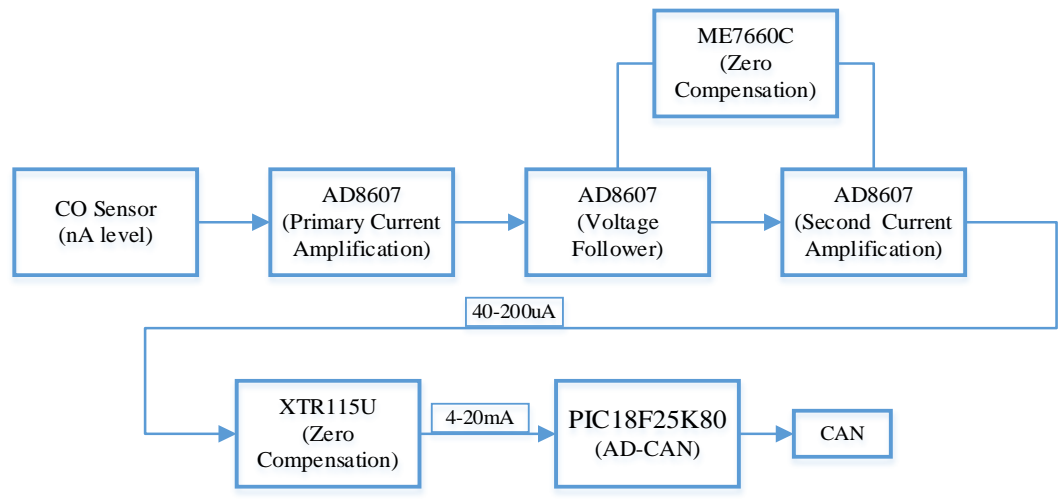

Fig. 2. CO acquisition part schematic diagram

As figure 2 shown, CO sensor output is 100nA level current signal. If it becomes a standard signal that can be acquired by $\mathrm{A} / \mathrm{D}$, it needs to design the conditioning 
circuit to amplify the weak current signal. The AD8607 chip is dual micropower rail-to-rail input and output amplifiers, feature low offset voltage as well as low input voltage and current noise, is selected as CO conditioning circuit. As the amplifier chip offset voltage is low, when the sensor is not zero, the error caused by the amplifier can be reduced to minimum. In order to make the nA-level current amplifying into uA level current, a two-stage current amplification and a voltage follower are used to make current to reach 40-200uA. The XTR115 precision current output transmitter chip is selected to make the current of 40-200uA into a standard industrial 4-20mA signal. The XIR115 chip provides a 5V reference voltage that powers the two AD8607 chips. The overall circuit does not need to be connected to other power supply chips, which reduces the unnecessary power consumption and made the circuit low power consumption and small heat. So it can be long-term uninterrupted working. Because current through multi-level and stage amplify have some slight perturbation, the voltage reversal chip ME7660C is selected to provide $\pm 5 \mathrm{~V}$ voltage. A $-5 \mathrm{~V}$ reference voltage is used to design a number of compensation circuit for the better output current. The circuit is completely isolated between the zero calibration and the range setting. Figure 3 shows the schematic diagram of the CO conditioning circuit.

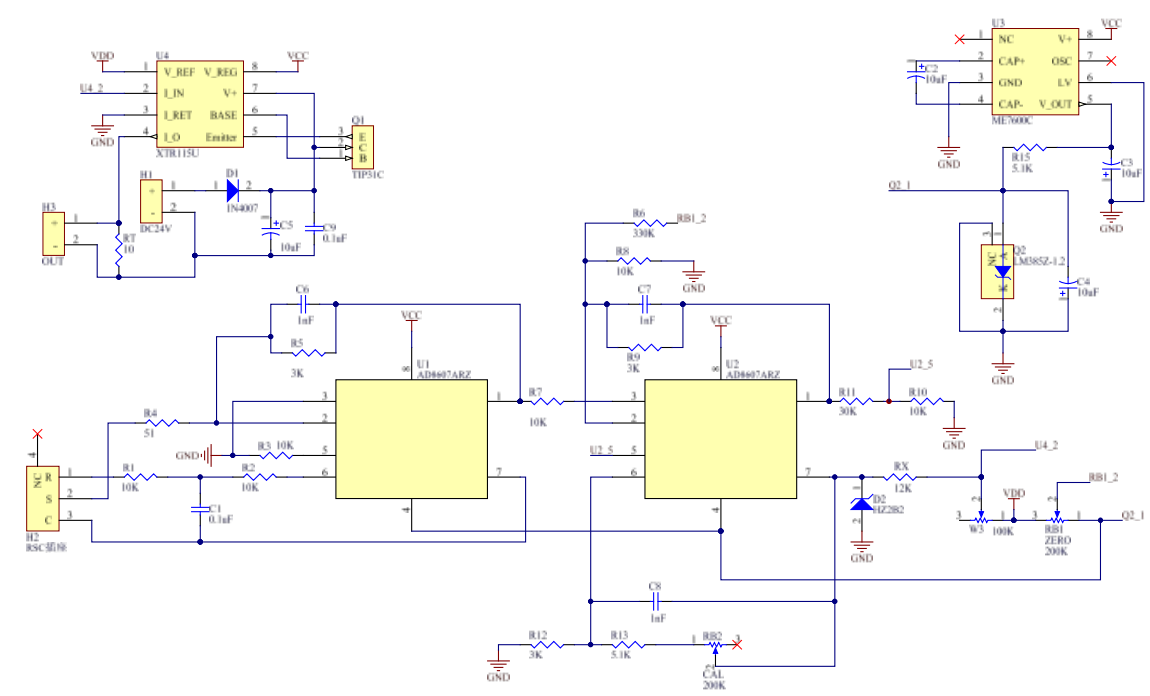

Fig. 3. CO conditioning circuit diagram

As Figure 4 shown, A/D acquisition and CAN transceiver module is based on PIC microcontroller design. PIC microcontroller CPU RISC structure is a streamlined instruction set. Harvard dual bus structure with instruction cycle of $160 \sim 200 \mathrm{~ns}$ runs fast .It can make the program memory access and data memory access parallel processing. This instruction pipeline structure is high efficiency. In addition, it also has a low operating voltage, low power consumption, and driving ability and so on. PIC series of its A / D 10-bit could meet the accuracy requirements. It has online debugging and programming (ISP) function. PIC18F25K80 is selected as PIC microcontroller. The model has ECAN bus module and 12-bit A / D acquisition module. The TJA1050 is an interface between the CAN protocol controller and the physical bus, and is a standard high-speed CAN transceiver. The TJA1050 provides 
differential transmit performance for the bus, providing differential receiver performance for the CAN controller. The PIC18F66K80 family of devices includes an enhanced controller area network and ECAN module. The CAN module is a serial interface for communicating with other peripherals or microcontroller devices. This interface or protocol is designed to allow communication in a noisy environment. The ECAN module is a communication controller that implements the CAN 2.0A or B protocol defined in the BOSCH specification. The module will support CAN 1.2, CAN 2.0A, CAN 2.0B Passive and CAN 2.0B Active version of the agreement. The Analog-to-Digital (A / D) converter module in the PIC18F66K80 family of devices has eight inputs (for 28-pin devices) and 11 inputs (for 40/44 and 64-pin devices). Here the chip ANO for the data input channel is used, the CO gas sensor conditioning circuit outputs $4-20 \mathrm{~mA}$ through the $200 \Omega$ resistor into a $0.8-4 \mathrm{~V}$ voltage signal. After voltage signal through A/D acquisition, the PIC18F25K80 gets real-time data of CO concentrations, and sends data on the CAN bus.

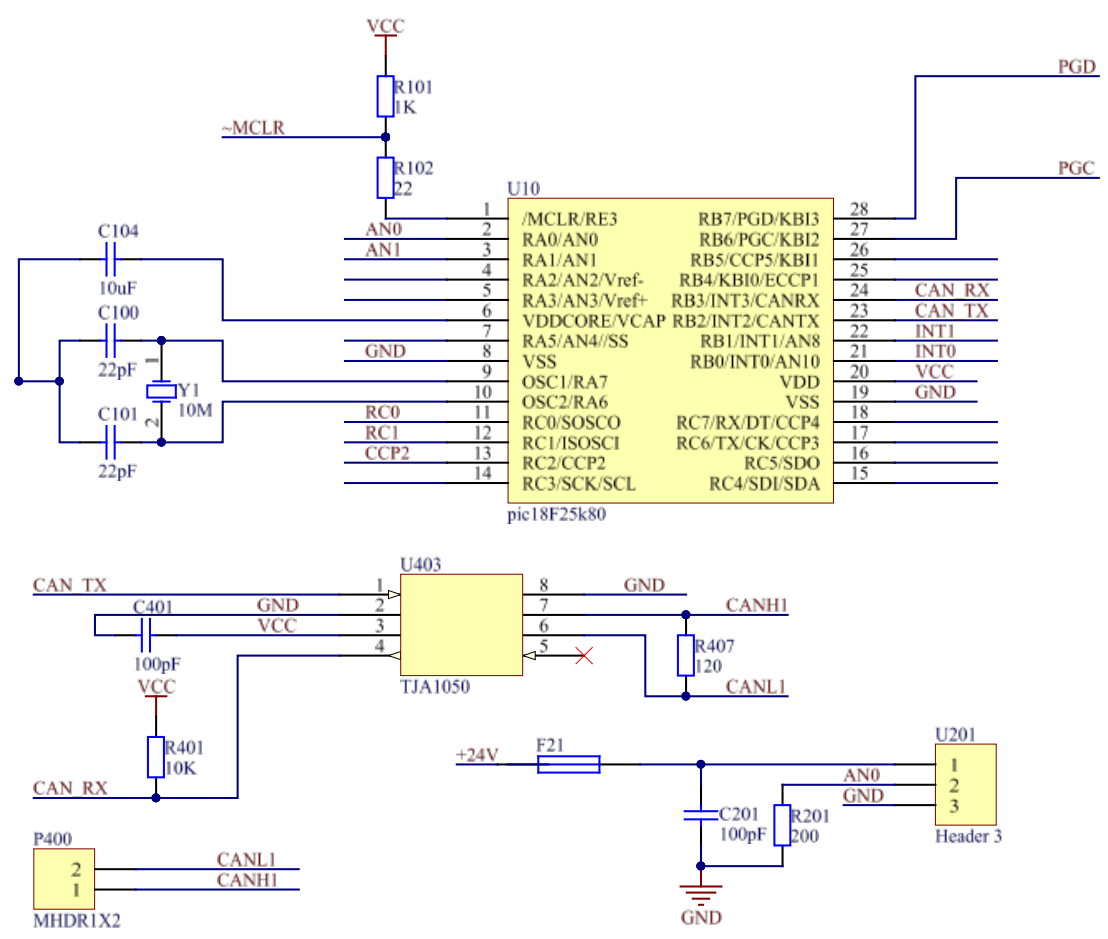

Fig. 4. CO A / D-CAN part of the circuit diagram

\subsubsection{Temperature and Humidity}

The SHT21 humidity and temperature sensor set new standards in terms of size and intelligence. It embeds in a reflow solderable Dual Flat No leads (DFN) package of $3 \times 3 \times 1.1 \mathrm{~mm}$ foot print, and provides calibrated and linearized signals in digital I2C format. With CMOSens ${ }^{\circledR}$ chip, the performance of reworked capacitive type humidity sensor and improved band gap temperature sensor has been lifted even beyond the outstanding level of the previous sensor generation. For example, measures have been taken to stabilize the behavior at high humidity levels. 
Furthermore, the resolution of SHT21 can be changed by command (8/12bit up to12/14bit for RH/T), low battery can be detected, and a checksum helps to improve communication reliability. SHT21 features a generation 4C CMOSens ${ }^{\circledR}$ chip. Besides the capacitive relative humidity sensor and the band gap temperature sensor, the chip contains an amplifier, A/D converter, OTP memory and a digital processing unit.

In summary, the functional and environmental requirements are fully satisfied. At the same time the whole temperature and humidity sensor will be treated with double waterproof, aiming to thoroughly anti-condensation damage. Waterproof of copper particles was used to make sheath. In the case of large humidity, when the ambient temperature decreases, the water molecules inside the sheath will condense to form water. If the water droplets attached to the circuit board, it may make the circuit board electrophoresis damage. The probe on the circuit board was used special potting material to pot. So it can work for a long time and do not need to be demarcated.

This circuit is designed using the Master Synchronous Serial Port (MSSP) module of the PIC28F25K80 microcontroller for communication with the SHT21 sensor. The MSSP module is a serial interface for communicating with other peripherals or microcontroller devices. These peripherals include serial EEPROM, shift register, display driver and A / D converter. The MSSP module can operate in two modes of Serial Peripheral Interface (SPI) and Internal Interconnect Module (I2C). The PIC28F25K80 microcontroller uses the MSSP module to receive the data sent by the SHT21 sensor via the IIC, then uses the RB2 / CANTX and RB3 / CANRX pins through the ECAN module to match the TJA1050 chip, and send the data to the CAN bus (Figure 5).

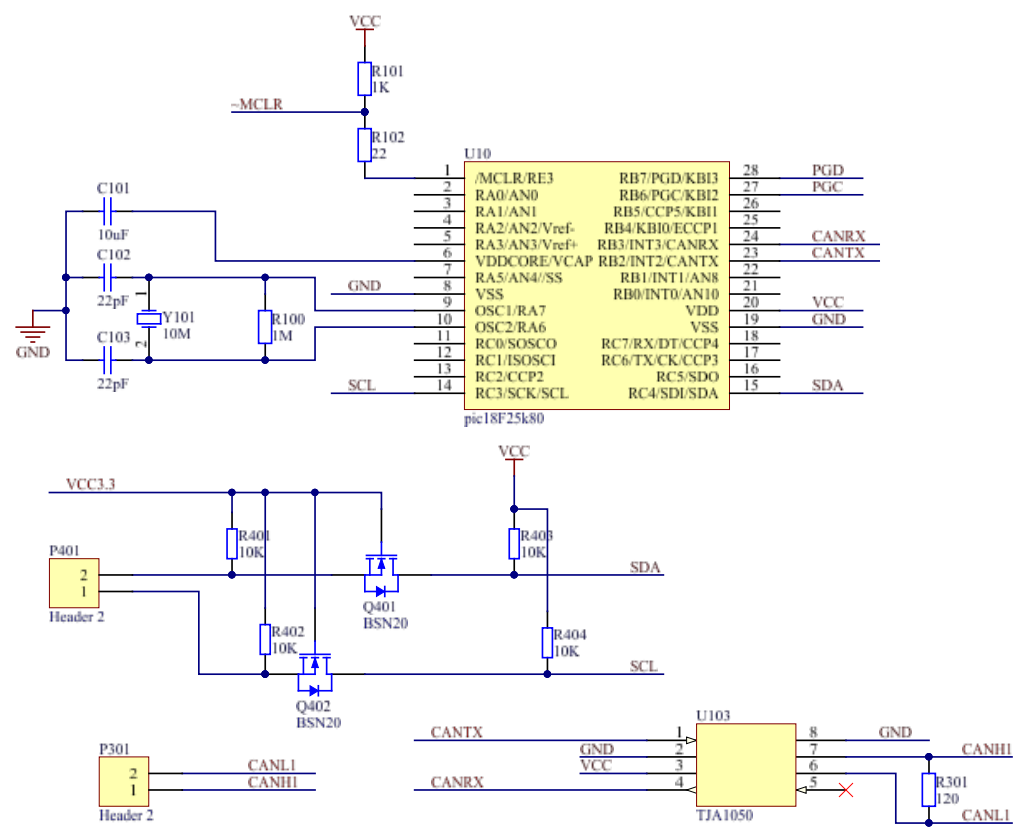

Fig. 5. SHT21 IIC-CAN circuit diagram 


\subsection{Data Query and Forwarding Module}

Figure 6 shows the ZigBee main node (Coordinator) function. The query module query and collect the data of the sensor array connected on CAN bus, and the data will be sorted and packaged. The ZigBee wireless transmitting module will sent the data to the host computer ZigBee receive port. The design of CAN receive module is based on the PIC microcontroller, in addition to carrying the above TJA1050 highspeed CAN transceiver chip and max232ewe+ chip, working temperature of $-40^{\circ} \mathrm{C}$ $\sim+85^{\circ} \mathrm{C}$ meets the temperature requirements of cotton pile site. MAX232EWE+ Chip is a single power level conversion chip designed by MAXI Company for RS-232 standard serial port. Because of using $+5 \mathrm{~V}$ single power supply, the device is particularly suitable for battery power supply system. Due to its low-power-off mode can reduce power consumption to 5uW, 2 RS-232C drivers are integrated inside, high integration, only 4 pieces of capacitance can work at a minimum. At the same time, the output port RS232 serial port connects to ZigBee serial port, sorts and packages the data received by CAN and sent out, transfers to the host computer ZigBee receiving port.
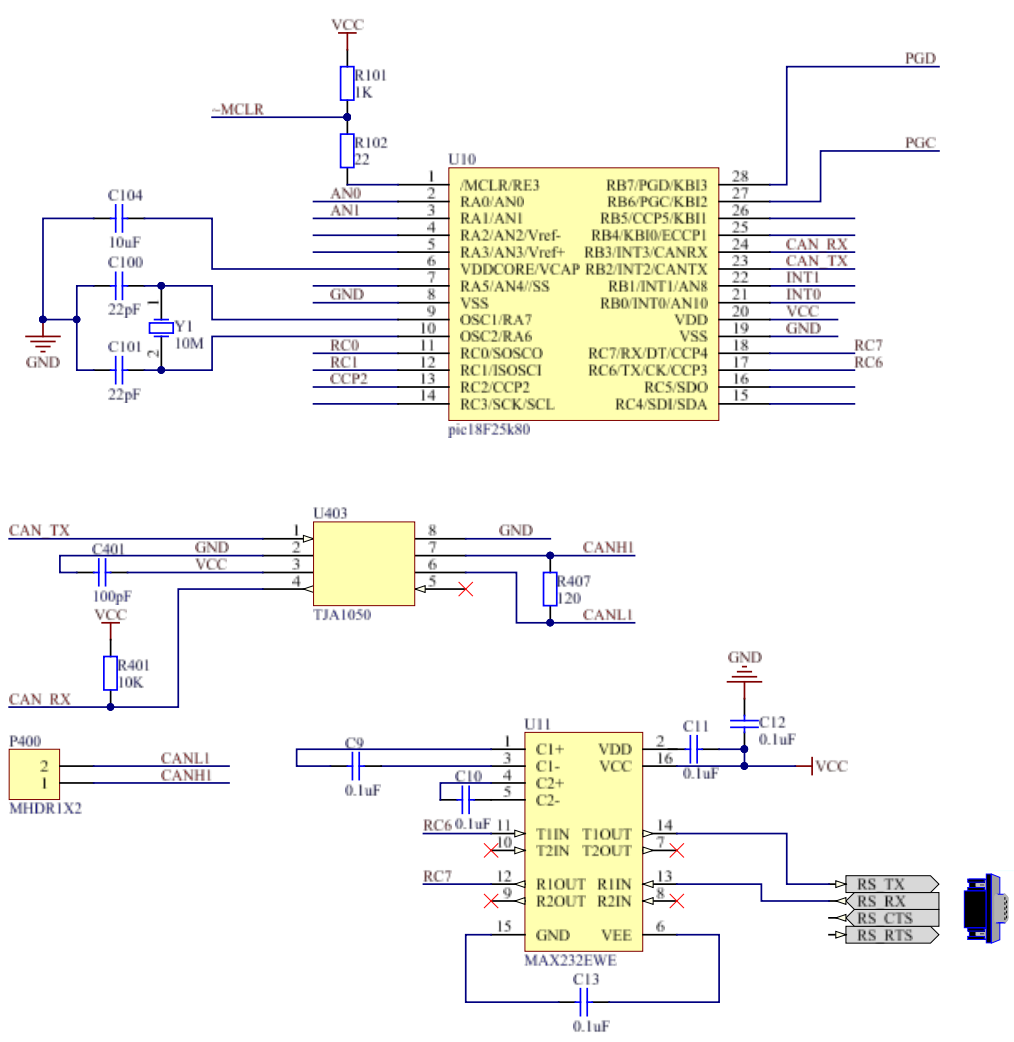

Fig. 6. Data query and forwarding module circuit diagram

\subsection{Data Receiving and Display Module}


PC program is prepared using graphical programming language LabVIEW, which provides five serial communication nodes of serial port early set, serial write, serial read, detect the number of bytes in the serial input cache, serial interrupt, respectively. Before serial communication between PC and wireless acquisition module, the serial port is configured firstly. It is also named serial port initialization, so that the computer serial port settings and wireless transceiver module serial port parameters keep consistent and correct communication.

The serial port of the Zigbee receiving module to the USB serial port is connected, and the NI-VISA driver from LabVIEW is used to introduce its data stream to complete the data reception. The program interface is designed to analyze the data and draw, and finally display it to the interface. When the data received exceeds the set threshold, the software interface emits images and sound alarms. The interface is as figure 7 shown.

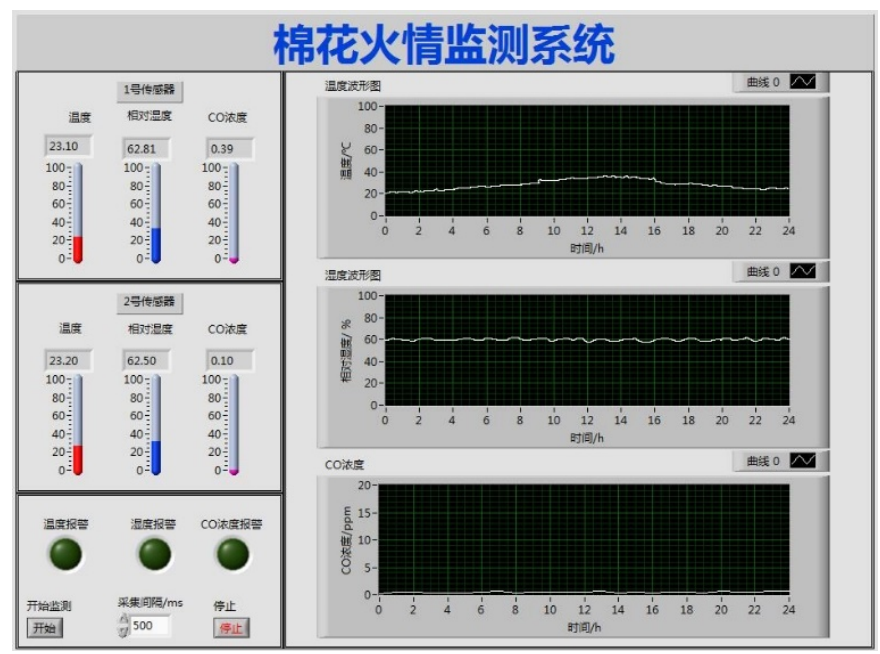

Fig. 7. PC software interface

\section{$4 \quad$ Fire Monitor System Experiments}

In order to evaluate the working performance of sensor, the following CO concentration, temperature and humidity acquisition experiments were taken.

\subsection{CO concentration experiment}

In a $30 \times 25 \times 17 \mathrm{~cm}$ rectangular box, $3 \mathrm{~g}$ wet unginned cotton was fired to simulate smoldering. The CO sensor was installed in the box, and collect data under the closed environment, with the acquisition time interval of 500ms and acquisition times of 2000. The 1000 sets of data were selected for analysis. The real-time data was stored in a txt file, in order to analyze data and find an appropriate filtering method, MATLAB software was used in this paper. 

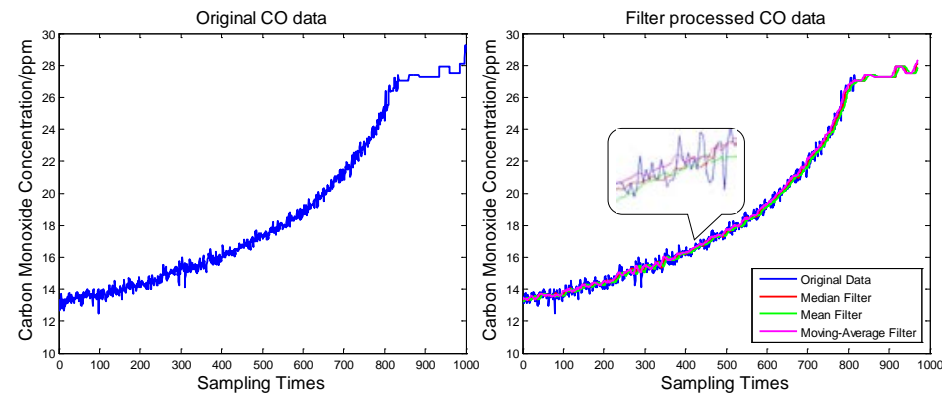

Fig. 8. CO sensor acquisition and filtering

Figure 8 shows data fluctuations of $\mathrm{CO}$ sensor during data collection. The data curve collected by CO sensors indicates that the CO sensor is sensitive. In this paper, three commonly used filters, including mean filter, median filter and movingaverage filter, were used to process data. The results show that the median filter error is the smallest when the window value is 9 , showing the best filtering result. The smoothing of moving-average filter method is the best, but the error is relatively large. Therefore, this paper uses the median filter method to deal with CO sensor data.

\subsection{Temperature experiment}

In total, $600 \mathrm{ml}$ hot water of $52.5^{\circ} \mathrm{C}$ was added into a glass container with a diameter of $12 \mathrm{~cm}$. The temperature sensor was sealed and put into the hot water. The system was used to collect temperature data. The system sets the acquisition time interval of 5000ms and acquires times of 550 .
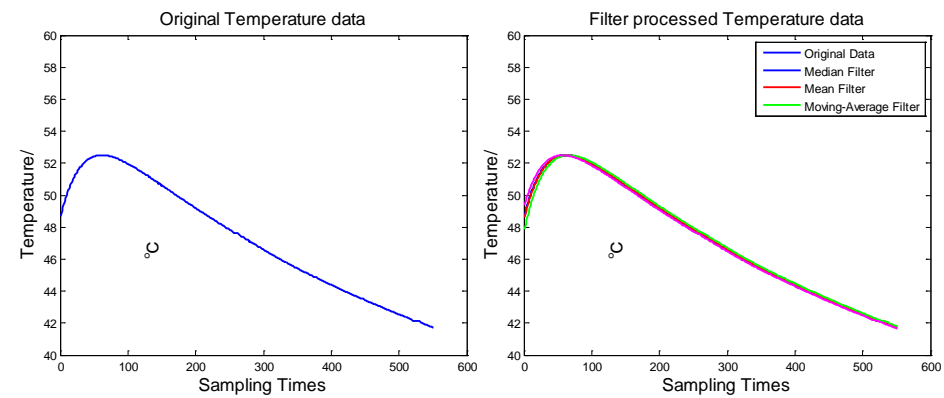

Fig. 9. Temperature sensor acquisition and filtering

Figure 9 shows the data acquired by temperature sensor, which presenting a smooth curve. The difference between raw data and filtering data is small. Because the filtering will occupy the hardware computing resources, with the small changes contributed by filtering, the temperature data could be directly used without filtering. Therefore, the system developed in this paper did not use the filter for the temperature sensor.

\subsection{Relative air humidity experiment}


In a 30x25x17cm rectangular box, a humidifier was placed in the upper left corner, and the humidity sensor was placed in the lower right corner. Firstly, the box was closed, then the humidifier was opened. The relative air humidity in the initial environment was $68 \%$. The system sets the acquisition time interval of $2000 \mathrm{~ms}$ and acquire times of 270 .
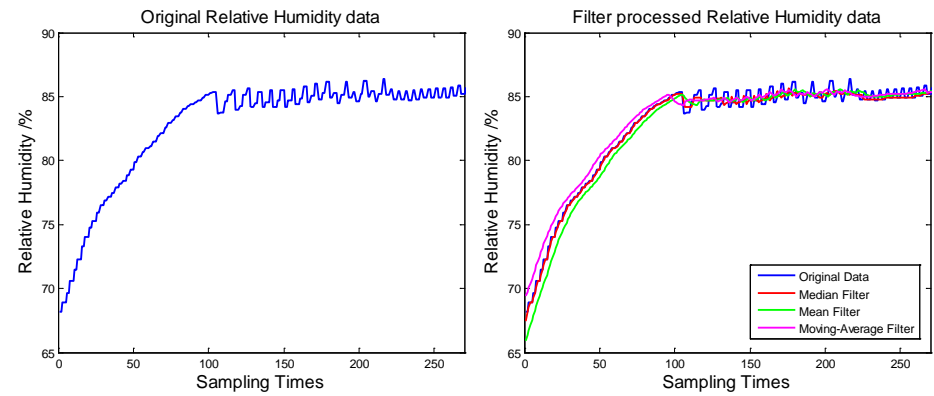

Fig. 10. Relative air humidity acquisition and filtering

Figure 10 shows that the humidity data curve is smooth, when air humidity rise in the box. However, the humidity data fluctuates, when the curve keep flat. The results show that the median filter error is the smallest, when the window value is 9, showing the best filtering result. Therefore, this paper used the median filter method to deal with relative air humidity sensor data.

\subsection{Cotton smoldering test}

In a $30 \times 25 \times 17 \mathrm{~cm}$ rectangular box, $50 \mathrm{~g}$ of wet unginned cotton was fired to simulate the smoldering. The CO sensor, temperature and humidity sensor were installed in the box. The data was collect in the closed box. The system set the acquisition time interval of $2000 \mathrm{~ms}$ and acquires times of 1200 . The real-time data was stored in a txt file. In order to analyze the data and find the appropriate filtering method, this paper used MATLAB software to analyze the data.
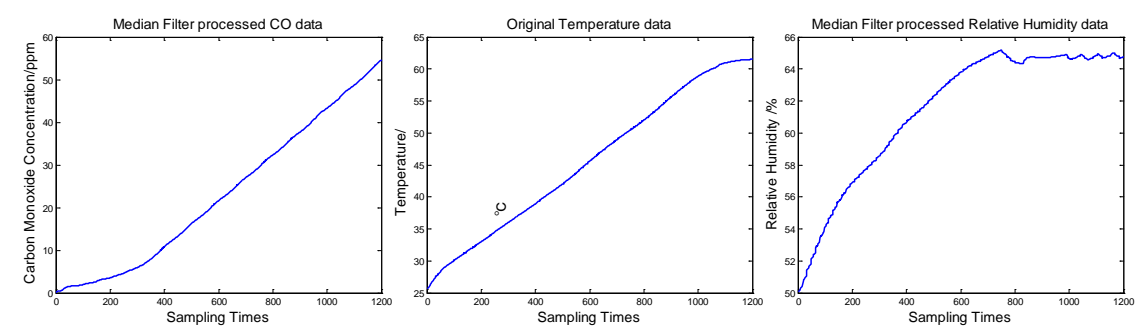

Fig. 11. The experimental curve of cotton smoldering

Figure 11 shows that, the sensing module can accurately measure the whole state of the wet unginned cotton smoldering. Smoldering monitoring is achievable. The selection of filtering algorithm is suitable. 


\section{Conclusions}

The unginned cotton smoldering, compared with the fire, produces more CO gas. Temperature and relative air humidity increase abnormally in the smoldering area. The collected data curve indicates that the CO sensor is sensitive. Because of the strong fluctuation, data collected by CO sensors must be filtered. When the window value is9, the median filtering is the best by comparing to the mean filter and the moving average filter. The temperature sensor's acquisition curve is smooth and does not need filtering. The rise of the humidity curve is smooth, and the flat section shows fluctuates. The results show that the median filter error is the smallest, when the window value is 9 , showing the best filtering result. Cotton pile fire monitoring system can monitor the $\mathrm{CO}$ concentration, temperature and humidity inside of unginned cotton pile. This system can warn in the early stage before fire. Multisensor array based fire monitor can provide the data of temperature, humidity and $\mathrm{CO}$ concentration. It is essential to effectively measure abnormal or normal situation of the cotton pile, and can make early warning for the cotton fire and reduce the loss of cotton fire disaster.

\section{Acknowledgments}

The study was supported by national science and technology support program of China (2015BAD19B04) and S\&T Nova Program of Beijing (Z1511000003150116).

\section{References}

1. Qixiang Zhu: The significance of the research on the automatic monitoring technology of temperature and humidity of cotton pile (in Chinese). J. China Cotton Processing. 02:23-25 (2010)

2. Mingzhu Zhang, Yihuang Shi: To carry out the temperature and humidity detection of cotton pile to solve the problem of stacking (in Chinese). J. China Cotton Processing. 02:22-23 (2011)

3. Tengda Cong, Za Can, Ruoyu Zhang, Honglei An, Zhangtao Ying, Xiangliang Li.: MoIlitoring and Mmanagement System of Temperature and Humidity for Cotton piles (in Chinese). J. Journal of Agricultural Mechanization Research. 01:193-196+200 (2013)

4. Wakelyn P J, Bertoniere N R, French A D: Cotton fiber chemistry and technology. M. CRC Press. (2006)

5. Hagen B C, Frette V, Kleppe G: Effects of heat flux scenarios on smoldering in cotton. J. Fire Safety Journal. 61: 144-159 (2013)

6. Hagen B C, Frette V, Kleppe G: Onset of smoldering in cotton: Effects of density. J. Fire Safety Journal. 46(3): 73-80 (2011) 
7. Chang L, Die M, Rongkun P: The Effect of Sample Size on Smoldering and the Transition to Flaming Combustion. C. //2011 Third International Conference on Measuring Technology and Mechatronics Automation. IEEE. 3: 793-797 (2011)

8. Jinjun Wang, Yi Zeng, Wei Yuan, Ran Tu, Yifei, Fangun Shi, Yongming Zhang: Early smoldering characteristics of cotton block and correlative compound aspirating smoke detection (in Chinese). J. Journal of Safety and Environment. 02:205-208 (2012)

9. Ruoyu Zhang, Tengda Cong, Za Kan: Design of Wireless Monitoring System for Temperature and Humidity of Cotton (in Chinese). J. China Cotton Processing. (3):1719 (2011)

10. Wen X: Research and design of distributed carbon monoxide (CO) concentration online detection system. C.//Intelligent Information Technology Application, 2008. IITA'08. Second International Symposium on. IEEE. 2: 829-833 (2008)

11. Ahriman N, Yulianto A, Kusriyanto M: Wireless sensor network application for carbon monoxide monitoring. C. //2015 9th International Conference on Telecommunication Systems Services and Applications (TSSA). IEEE. 1-4 (2015)

12. Enliang Xia, Shenyou Liu, Xudong Chen, Yanan Hou: A comparative study on the characteristic of cotton smoldering and flaming combustion (in Chinese). J. Fire Safety Science. 02:70-76 (2013)

13. Yu Hu, Zhibing Wang: Experimental study on the smoldering characteristics of lint cotton and unginned cotton (in Chinese). J. Fire Safety Science. (1):47-51 (2015)

14.ZHAO Yue, PIAO Renguan, WANG He, XU Zhijun: Multi-channel Gas Detection System Design Based on LabVIEW (in Chinese). J. Instrument Technique and Sensor.(4):37-40 (2013)

15. Xiaoping Ding: Design and Research of CO Detection and Alarm System Based on Wireless Network (in Chinese). J. Computer Knowledge and Technology. (13):31323134 (2014)

16. WANG Xiaozeng, ZENG Hui, YANG Jiuhong: Temperature Self-compensation Realization and Hardware Circuit Design of CO Detection. J. Instrument Technique And Sensor. (11):69-71 (2007)

17. Wang W, Peng Y, Peng Y: Temperature and Humidity Detection System Based on ZigBee Protocol. J. Metallurgical \& Mining Industry. (3) (2015)

18. Barroca N, Borges L M, Velez F J: Wireless sensor networks for temperature and humidity monitoring within concrete structures. J. Construction and Building Materials40: 1156-1166 (2013)

19. Yi C, Bai F M.: ZigBee-based CC2530 Granary Temperature and Humidity Monitoring System. J. Journal of Changchun University of Science and Technology (Natural Science Edition). 4: 016 (2011)

20. Hao Cai, Yagang Wang, Kai Wang: Design of Industrial Wireless Temperature and Humidity Detection Terminal Based on Modular ZigBee (in Chinese). J. Instrument Technique and Sensor. (5):38-39,94. (2013) 